hep-ph/yymmmmm

CTEQ/PUB/02

FNAL/

$\mathrm{PSU} / \mathrm{TH} / 136$

\title{
Measuring Parton Densities in the Pomeron
}

\author{
John C. Collins ${ }^{a}$, Joey Huston ${ }^{b}$, Jon Pumplin ${ }^{b}$, Harry Weerts ${ }^{b}$, and J.J. Whitmore ${ }^{a}$ \\ ${ }^{a}$ Department of Physics, Penn State University, University Park, PA 16802, U.S.A. \\ ${ }^{b}$ Physics and Astronomy Department, Michigan State University, East Lansing MI 48824, U.S.A.
}

(December 8, 1994)

\begin{abstract}
We present a program to measure the parton densities in the pomeron using diffractive deep inelastic scattering and diffractive photoproduction, and to test the resulting parton densities by applying them to other processes such as the diffractive production of jets in hadron-hadron collisions. Since QCD factorization has been predicted not to apply to hard diffractive scattering, this program of fitting and using parton densities might be expected to fail. Its success or failure will provide useful information on the space-time structure of the pomeron.
\end{abstract}

12.38.Bx, 12.38.Qk, 12.40.Nn, 13.60.Hb, 13.85.Ni, 13.87.-a

Typeset using REVTEX 


\section{INTRODUCTION}

A spectacular effect in very high energy hadron collisions is that of diffraction, wherein a hadron can scatter and emerge unscathed, with only a small deflection and loss in longitudinal momentum. The rest of the final state continues to exhibit the usual fragility of hadrons in a collision where the total energy is far greater than all intrinsic hadronic energy scales. There is an old and established quantitative phenomenology that describes diffraction and the bulk of the cross section. This is the theory of Reggeons and the pomeron [1]. Unfortunately the understanding of the pomeron in terms of the fundamental theory of QCD is weak.

With the advent of high energy colliders, and detectors with a wide rapidity coverage, it is now possible to study diffractive collisions that contain a hard scattering. Such 'hard diffractive scattering' provides a tool whereby we can achieve a better understanding of the pomeron at the fundamental level.

In the model of Ingelman and Schlein [2], hard diffraction is treated by considering the exchanged pomeron as an almost real particle. This is entirely similar in spirit to the way low $Q^{2}$ electroproduction is used as a way of obtaining collisions with almost real photons. However, Collins, Frankfurt and Strikman [3- 5] (CFS) have questioned the validity of QCD factorization in this case, and have given a specific mechanism for the breakdown of factorization. Their mechanism, 'coherent hard diffraction', has the signature that there will be events where the hard scattering takes almost all the momentum of the pomeron. Recent UA8 data [6] appear to possess this property. On the theoretical side, Levin and collaborators [7] have also verified that QCD predicts that factorization should not apply to hard diffraction with two incoming hadrons, and a calculation by Berera and Soper 8 comes to the same conclusion as CFS.

We therefore propose a program to measure the parton densities in a pomeron. Because the pomeron is an isosinglet and is self-charge-conjugate, the number of independent distributions to be measured is much smaller than for the proton. We will show that the program can therefore be carried out with existing detectors. Two processes suffice to fix the parton densities, and a third process will serve to test factorization. See also a recent paper by Ingelman [9]. Many of the individual points in this paper are already known to experts in the field [10]. Here we are showing the need for a coordinated program of measurements involving different experiments.

If the UA8 results [6] are indeed evidence of the CFS mechanism [3], there will be a quite spectacular failure of the program of fitting parton densities. As CFS show, this is a consequence of a failure of the applicability of the factorization theorem, which is in turn a direct consequence of the space-time structure of the pomeron. Therefore, the success or failure of the fitting will rather directly fulfill the promise of hard scattering to turn a microscope onto the strong interaction.

Although the measurement of classical diffraction requires "Roman Pot" detectors in the beam pipe, we will show that a substantial and interesting part of our program can be

carried out without them. One can instead use a rapidity gap signature, thanks to the large rapidity coverage of current detectors (CDF and D0 at the Fermilab $p \bar{p}$ collider, H1 and ZEUS at the DESY $e^{-} p$ collider). Indeed, ZEUS has already reported data [11] on events with hard scattering in conjunction with rapidity gaps of this kind. These data are quite 
suggestive of pomeron exchange. In addition, the D0 experiment has reported [12 on a search for jet production with rapidity gaps between the jets; their data are suggestive of the presence of such gaps. CDF [13] has also found evidence for such gaps.

The outline of this paper is as follows. In Sect. II we explain the processes that are of interest. In Sect. III we give the formulae for the cross sections for these processes within the Ingelman-Schlein model, and in Sect. [V] we show how to use them to measure the parton densities in the pomeron. In Sect. $\nabla$ we show how measurements can also be made with present detectors using a rapidity gap signature. Then in Sect. V1 we summarize the consequences of the CFS [3] result for our fitting program. Some remarks on experimental details are given in Sect. VII. In Sect. VIII, we summarize the processes that should be investigated.

\section{THE PROCESSES}

We consider the following types of processes:

1. Diffractive deep inelastic $e-p$ scattering: $e+p \rightarrow e+X+p$.

2. Diffractive deep inelastic $e-p$ scattering, with the measurement of jets in the final state, e.g., $e+p \rightarrow e+J_{1}+J_{2}+X+p$.

3. Diffractive photoproduction of jets, with the jets being produced by the direct photon process: $\gamma+p \rightarrow J_{1}+J_{2}+X+p$.

4. Diffractive photoproduction of jets via a resolved photon process, i.e., one with a photon remnant jet (a photon beam jet).

5. Diffractive hadroproduction of one or more jets: $p+\bar{p} \rightarrow J_{1}+\ldots+X+\bar{p}$.

6. Other hard diffractive scattering in hadron-hadron collisions, e.g., production of the $W$ or the $Z: p+\bar{p} \rightarrow W / Z+X+\bar{p}$, and production of direct photons at large transverse momentum: $p+\bar{p} \rightarrow \gamma+X+\bar{p}$.

In each of these processes we can allow for different numbers of jets than those explicitly indicated. In each case the explicit final-state $p$ (or $\bar{p}$ ) is a small angle diffracted proton (or anti-proton) that carries $\gtrsim 95 \%$ of the momentum of the incident $p$ (or $\bar{p}$ ). The generalization in which the diffracted particle is replaced by a low-mass inelastically diffracted state will be discussed in Sect. $\nabla$.

For the sake of definiteness, the following discussion of the kinematics will be presented for the case of hadron-hadron scattering. Only minor changes are required for the other processes.

In terms of the momenta $p_{1}$ and $p_{2}$ of the incoming hadrons, the diffracted hadron's momentum is

\footnotetext{
${ }^{1}$ By 'jet', we will generally mean a jet that originates from the hard scattering, rather than a beam jet.
} 


$$
p_{2}^{\prime \mu}=\left(1-x_{\mathbb{P}}\right) p_{2}^{\mu}+\epsilon p_{1}^{\mu}+p_{T}^{\mu},
$$

with $x_{\mathbb{P}} \ll 1$ denoting the fraction of the diffracted hadron's momentum transferred by the exchanged pomeron to the rest of the final state, and $p_{T}^{\mu}$ denoting the transverse momentum of the diffracted hadron. The quantity $\epsilon$ is very small $(\mathcal{O}(1 / s))$, and the square of the invariant momentum transfer is

$$
t=\left(p_{2}^{\prime}-p_{2}\right)^{2}=-\left(\left|p_{T}\right|^{2}+x_{\mathbb{P}}^{2} m_{2}^{2}\right) /\left(1-x_{\mathbb{P}}\right) \approx-\left|p_{T}\right|^{2} .
$$

For pomeron exchange to dominate, it is generally assumed that one needs

$$
x_{\mathbb{P}} \lesssim 0.05
$$

Our definition of the pomeron is essentially kinematic: it is whatever exchanged object gives the dominant behavior at small $x_{\mathbb{P}}=1-x$, where $x=p_{2}^{\prime+} / p_{2}{ }^{+}$is the fraction $(\approx 1)$ of the large light-cone momentum kept by the diffracted hadron. This is equivalent to defining the pomeron as the mechanism that produces rapidity gaps, since energy-momentum conservation implies a substantial rapidity gap $\left(\gtrsim \ln \left(1 / x_{\mathbb{P}}\right)\right)$ between the diffracted hadron and the rest of the final state whenever $x_{\mathbb{P}}$ is small.

Obvious graphs that should give leading twist contributions to these processes are illustrated in Fig. 1. In each case, we show a typical lowest order graph for the hard scattering (e.g., partons making jets), together with the pomeron exchange. These graphs represent the Ingelman-Schlein model, and the question we wish to suggest be experimentally studied is whether the quantitative phenomenology represented by that model is in fact correct.

\section{INGELMAN-SCHLEIN MODEL}

\section{A. Jets in diffractive hadron-hadron scattering}

Within the Ingelman-Schlein model, the cross section for diffractive jet production in hadron-hadron collisions is

$$
\begin{array}{r}
\frac{d \sigma\left(A+B \rightarrow J_{1}+\ldots+B\right)}{d t d x_{\mathbb{P}}}=\frac{N}{16 \pi}\left|\beta_{B \mathbb{P}}(t)\right|^{2} x_{\mathbb{P}}^{1-2 \alpha_{\mathbb{P}}(t)} \sum_{a, b} \int d x_{a} \frac{d x_{b}}{x_{\mathbb{P}}} \\
\times f_{a / A}\left(x_{a} ; \mu\right) f_{b / \mathbb{P}}\left(x_{b} / x_{\mathbb{P}}, t ; \mu\right) \hat{\sigma}\left(a+b \rightarrow J_{1}+\ldots\right) .
\end{array}
$$

Here, $\mu$ is the usual renormalization and factorization scale, which we will assume to be in the $\overline{\mathrm{MS}}$ scheme, $f_{b / \mathbb{P}}\left(x_{b} / x_{\mathbb{P}}\right)$ is the parton density in the pomeron, $f_{a / A}\left(x_{a}\right)$ is the parton density in the non-diffracted hadron $A$, and $\hat{\sigma}$ is the usual hard scattering cross section. The last two quantities are identical to the corresponding quantities used in hard non-diffractive

\footnotetext{
${ }^{2}$ We define light-cone coordinates for any vector $V^{\mu}$ by $V^{ \pm}=\left(V^{0} \pm V^{z}\right) / \sqrt{2}$ and $V^{T}=\left(V^{x}, V^{y}\right)$ in a frame in which the incoming particle $p_{1}$ is moving in the $-z$ direction and $p_{2}$ is moving in the $+z$ direction. See App. A for more details. Our $z$ axis is the same as in ZEUS, when $p_{2}$ is the proton.
} 
scattering, so we consider them known. The hard scattering cross section can be differential in the jet variables, or integrated over a suitable range. It can be applied to any desired number of explicitly detected jets.

A tricky point is the normalization. In view of the significance of the test of the momentum sum rule for the parton densities in the pomeron, this is important to get correct. Unfortunately there is controversy on the issue. In Eq. (4), we have used the normalization factor from [2, 17], but have introduced a constant $N$ to allow for the possibility of a change. In any case, the idea is that the exchanged pomeron is to be thought of as lying on a Regge trajectory, and thus is an analytic continuation from particle poles in the region of $t \geq 0$. The existence of a momentum sum rule for a real particle is unambiguous; the controversy is about the treatment of the analytic continuation.

Ingelman and Schlein [2] would set

$$
N=1 \text { (Ingelman and Schlein). }
$$

Their argument relies on a formula given by Field and Fox [18 for the pomeron-proton total cross section. This formula, in effect, defines what a properly normalized pomeron state would be. The same formula for the pomeron-proton cross section is given, for example, by Kaidalov [19], and we believe it is to be regarded as the accepted formula in Regge theory.

Landshoff [15] argues that a more appropriate normalization would be one that works if the pomeron exchange is replaced by a photon. His argument leads to

$$
N=\frac{2}{\pi} \text { (Donnachie and Landshoff). }
$$

We have another argument for the incorrectness of Eq. (5). We argue that we should also be able to apply the Regge formula for diffraction when the exchanged pomeron is replaced by a Regge trajectory on which there is a massless spin-0 particle. As we explain in App. Q, the normalizations are unambiguous and appear to give disagreement with the standard Regge formulae [18, 19.

At this point we find ourselves unable to resolve the disagreements. The cross section formulae in [18, 19] are given without proof, as if they are obvious; but we find the proof far from obvious. In any event, the normalization factor $N$ is common to all the formulae for diffractive scattering.

We now return to the physics that is unaffected by the normalization constant, which is everything but the momentum sum rule. The Ingelman-Schlein picture (Eq. (田)) assumes dominance by single pomeron exchange, which is a useful phenomenological approximation in other applications of pomeron physics [1, 14, 15. It also assumes the validity of factorization for hard scattering in pomeron-hadron collisions - the notion we wish to test.

The coupling $\beta_{B \mathbb{P}}(t)$ of the pomeron to the diffracted hadron, and the pomeron trajectory $\alpha_{\mathbb{P}}(t)$, can be obtained by fits to the elastic cross section at small $-t$ :

$$
\frac{d \sigma(A B \rightarrow A B)}{d t}=\frac{1}{16 \pi}\left|\beta_{A \mathbb{P}}(t)\right|^{2}\left|\beta_{B \mathbb{P}}(t)\right|^{2}\left(\frac{s}{s_{0}}\right)^{2 \alpha_{\mathbb{P}}(t)-2} .
$$

\footnotetext{
3 This represents a change from the preprint version of the paper.
} 
The pomeron coupling and its intercept also give total hadron-hadron cross sections:

$$
\sigma_{\text {tot }}(A B)=\beta_{A \mathbb{P}}(0) \beta_{B \mathbb{P}}(0)\left(\frac{s}{s_{0}}\right)^{\alpha_{\mathbb{P}}(0)-1}
$$

where we have omitted a signature factor that is reasonably close to unity.

With $s_{0}=1 \mathrm{GeV}^{2}$, we can use [1, 14]

$$
\beta_{p \mathbb{P}}(t)=\beta_{\bar{p} \mathbb{P}}(t) \simeq 4.6 \mathrm{mb}^{1 / 2} e^{1.9 \mathrm{GeV}^{-2} t},
$$

and

$$
\alpha_{\mathbb{P}}(t) \simeq 1.08+0.25 \mathrm{GeV}^{-2} t
$$

Then Eq. (4) can be used to measure the parton densities in the pomeron - or at least one combination of them. The momentum of the outgoing diffracted hadron gives both $x_{\mathbb{P}}$ and $t$. (A useful independent measure of $x_{\mathbb{P}}=M^{2} / s$ can also be obtained from the diffractively produced state, for events in which the edge of the rapidity gap appears inside the detector - see App. A.) Measurement of the two jets allows one to reconstruct, to a first approximation, the kinematics of the parton-parton collision. In particular, it gives the momentum fraction $x_{b} / x_{\mathbb{P}}$ of parton $b$ relative to the pomeron. QCD radiative corrections can be allowed for in the usual fashion: one must only remember that the collision is to be treated as a pomeron-hadron collision, whose energy will vary from event to event.

\section{B. Diffractive deep inelastic scattering and photoproduction}

Diffractive hadroproduction of jets by itself is not sufficient to extract the flavor-separated parton densities. We need to use more processes. It is very well established [1] from conventional Regge phenomenology that the pomeron is self-charge-conjugate and isoscalar. Thus the density of any flavor of anti-quark is equal to the density of the corresponding quark,

$$
f_{i / \mathbb{P}}(x)=f_{\bar{\imath} / \mathbb{P}}(x),
$$

and the densities of the up and down quarks and antiquarks are all equal:

$$
f_{u / \mathbb{P}}(x)=f_{d / \mathbb{P}}(x)=f_{\bar{u} / \mathbb{P}}(x)=f_{\bar{d} / \mathbb{P}}(x) \equiv f_{q / \mathbb{P}}(x) .
$$

To a good first approximation, there are therefore only two parton densities to measure: the gluon density $f_{g / \mathbb{P}}$ and the quark density $f_{q / \mathbb{P}}$. The strange quark density $f_{s / \mathbb{P}}=f_{\bar{s} / \mathbb{P}}$ is probably less important, but it could be measured using charged-current charm production at HERA, just as the strange quark density in nucleons is measured by dimuon production in neutrino scattering. The other quarks (charm and bottom) are generally considered heavy enough that their densities can be correctly generated dynamically by evolution from the known light parton densities, at least to an accuracy that is sufficient for our purposes.

Formulae similar to, but simpler than, Eq. (4) can be written for the diffractive deep inelastic structure functions and for the direct photoproduction of jets. If we measure the 
deep inelastic structure functions $F_{1}$ and $F_{2}$ differentially in the momentum of the diffracted hadron, they can be expressed in terms of the structure functions $F_{1}^{\mathbb{P}}$ and $F_{2}^{\mathbb{P}}$ for the pomeron:

$$
\begin{aligned}
& \frac{d F_{1}^{\text {diffractive }}\left(x_{b j}, Q ; t, x_{\mathbb{P}}\right)}{d t d x_{\mathbb{P}}}=\frac{N}{16 \pi}\left|\beta_{B \mathbb{P}}(t)\right|^{2} x_{\mathbb{P}}{ }^{-2 \alpha_{\mathbb{P}}(t)} F_{1}^{\mathbb{P}}\left(x_{b j} / x_{\mathbb{P}}, Q\right) \\
& \frac{d F_{2}^{\text {diffractive }}\left(x_{b j}, Q ; t, x_{\mathbb{P}}\right)}{d t d x_{\mathbb{P}}}=\frac{N}{16 \pi}\left|\beta_{B \mathbb{P}}(t)\right|^{2} x_{\mathbb{P}}{ }^{1-2 \alpha_{\mathbb{P}}(t)} F_{2}^{\mathbb{P}}\left(x_{b j} / x_{\mathbb{P}}, Q\right) .
\end{aligned}
$$

Note the different powers of $x_{\mathbb{P}}$ in the above equations! The pomeron coupling and trajectory function are given by the expressions in Eqs. (9) and (10). Ordinarily, the structure functions $F_{1}$ and $F_{2}$ are defined for totally inclusive deep inelastic scattering. But our diffractive cross section is actually semi-inclusive, so that there are extra structure functions. We explain these issues in App. B.

Similarly, for the direct photoproduction of jets we have

$$
\begin{aligned}
& \left.\frac{d \sigma\left(\gamma+B \rightarrow J_{1}+\ldots+p\right)}{d t d x_{\mathbb{P}}}\right|_{\text {direct }}=\frac{N}{16 \pi}\left|\beta_{B \mathbb{P}}(t)\right|^{2} x_{\mathbb{P}}{ }^{1-2 \alpha_{\mathbb{P}}(t)} \\
& \times \sum_{b} \int \frac{d x_{b}}{x_{\mathbb{P}}} f_{b / \mathbb{P}}\left(x_{b} / x_{\mathbb{P}}, t ; \mu\right) \hat{\sigma}\left(\gamma+b \rightarrow J_{1}+\ldots\right)_{\mathrm{direct}} .
\end{aligned}
$$

The formula for the resolved contribution to the photoproduction of jets is identical to that for hadroproduction, Eq. (4), with the parton densities in the hadron $A$ replaced by the parton densities in the photon.

It is also possible to consider the case where the photon is diffracted quasi-elastically into a $\rho^{0}$ meson, or to some other low mass state that carries $\gtrsim 90 \%$ of the original $\gamma$ momentum, with the corresponding pomeron undergoing hard scattering on the other incident particle. See [20] for some recent predictions.

\section{MEASURING THE PARTON DENSITIES}

For the sake of definiteness, we consider diffractive deep inelastic scattering (DIS) and diffractive direct photoproduction of jets as the processes to measure the parton densities of the pomeron. We then treat the other processes of Sect. II as providing a tests of factorization. This is reasonable, since the arguments against factorization apply at their strongest to hard diffraction in hadron-hadron collisions, and thus to production of jets both in diffractive hadron-hadron collisions and in diffractive resolved photoproduction.

With flavor-separated parton densities for the pomeron, one can test the momentum sum-rule:

$$
\sum_{i} \int_{0}^{1} d x x f_{i / H}(x)=1,
$$

as applied to the pomeron. In the case of parton densities in an ordinary hadron, this sum rule is a prediction (provided that a suitable definition of the parton densities is used, such

as the $\overline{\mathrm{MS}}$ definition). But it is by no means required that the sum rule hold for the parton densities in the pomeron [17, [15, ]]. The reason is that the proof of the momentum sum rule 
Eq. (16) requires one to take the expectation value of the energy-momentum tensor in the hadron state $H$. For the proof to apply to the pomeron, one would have to construct a quantum mechanical state for the exchanged pomeron in diffractive processes, and such a concept does not appear to exist.

Deep inelastic electron-proton scattering primarily measures the quark distribution:

$$
\begin{aligned}
F_{2}^{\mathbb{P}}(x) & =x \sum e_{i}^{2} f_{i / \mathbb{P}}(x)+\mathcal{O}\left(\alpha_{s}\right) \\
& =\frac{10}{9} x f_{q / \mathbb{P}}(x)+\frac{2}{9} x f_{s / \mathbb{P}}(x)+\mathcal{O}\left(\alpha_{s}\right) .
\end{aligned}
$$

The strange quark term in Eq. (17) is presumably substantially smaller than the up and down quark terms, and can be ignored in a first pass at a fit. However, the gluonic part of the $\mathcal{O}\left(\alpha_{s}\right)$ term is probably significant at small $x_{b j} / x_{\mathbb{P}}$.

To measure the strange quark distribution, one could investigate the charged current process with the production either of muons or of charm in the current fragmentation region:

$$
e+p \rightarrow \nu+\mu / D+X+p \text {. }
$$

Muons would typically have come from charm decay, and charm production is mostly from scattering off strange quarks, in charged current events.

Direct photoproduction of jets would be sensitive to both the quark and gluon densities. The version of Eq. (15) that applies to this case is, schematically,

$$
\left.\sigma(\gamma+p \rightarrow \text { jets }+X+p)\right|_{\text {direct }} \propto \alpha_{\mathrm{em}} \alpha_{s}\left(A \frac{10}{9} x f_{q / \mathbb{P}}+A \frac{2}{9} x f_{s / \mathbb{P}}+B x f_{g / \mathbb{P}}+\mathcal{O}\left(\alpha_{s}\right)\right),
$$

where $A$ and $B$ are known coefficients that carry the detailed kinematic dependence from the pomeron factors and the hard scattering coefficient $\hat{\sigma}$ in Eq. (15). The formulae for these coefficients are the same as for the same processes without the diffractive requirement. The photon does not have to be real: identical physics would be probed by DIS with an extra jet beyond the minimum current quark jet given by the parton model. The only penalty would be a loss of cross section. The simplest way to extract the parton densities in Eq. (19) would be to measure 2-jet cross sections, with the kinematics of the underlying photon-parton scattering being deduced by treating it as a $2 \rightarrow 2$ scattering.

Hence the diffractive subsets of the two processes, DIS and direct photoproduction of jets, will measure the parton densities. The results of a fit can then be put into Eq. (田) to predict diffractive cross sections for other hard processes, in particular hadroproduction of jets and of $W$ or $Z$ bosons at the Tevatron, and diffractive resolved photoproduction of jets at HERA. They can also be used to calculate the cross section that UA8 [6] have measured. If the UA8 results are interpreted according to the CFS mechanism [3], then a readily visible excess of events can be predicted at $x_{b} / x_{\mathbb{P}} \rightarrow 1$ for the hadron-hadron experiments relative to the DIS and direct photoproduction experiments. In any event the parton densities measured at HERA can be compared with those fitted by UA8 to their data, both in shape and in absolute normalization.

A full quantitative analysis and fit for the parton densities in the pomeron would require the use of the higher order corrections to the formulae for the cross sections and structure functions, such as the $\mathcal{O}\left(\alpha_{s}\right)$ terms in Eq. (17) and Eq. (19). These are standard known expressions. 


\section{RAPIDITY GAP SIGNATURE}

The ideal experimental signature of the pomeron for the purposes of deep inelastic physics in a colliding beam experiment involves observing a "quasi-elastically" scattered proton using a Roman Pot detector. These detectors should be located very close to the unscattered beam direction, because, in the region of pomeron-dominance, the quasi-elastic protons have longitudinal momenta $0.9-0.99$ or more times the incident proton momentum, and transverse momenta $\lesssim 1 \mathrm{GeV}$. It then follows by kinematics that there is a rapidity gap between the quasi-elastic proton and the rest of the event.f

Roman Pots have been added to the HERA detector ZEUS, but are not yet present in H1. They are also not present in either detector at the Tevatron, although CDF took some early data with pots, and has proposed adding new ones in an eventual upgrade. In all current experiments, there are several units of rapidity between the hadrons that can be detected by a Roman Pot detector and by the rest of the detector.

Fortunately, much of the same physics can be probed using a rapidity gap signature alone. The gap signature is defined by a requirement that no final state particles are produced in a sufficiently long region in pseudorapidity that extends from the edge of the main detector toward its center. The data from [11,21], together with standard pomeron phenomenology, suggest that "sufficiently long" can be read as "length $\gtrsim 3$ units" of (pseudo-)rapidity. The gap signature (for a proton beam) allows a mixture of (1) single "quasi-elastic" protons, (2) low-mass diffractive excitations of the proton, and (3) moderately high mass diffractive excitations, whose mass is nevertheless low enough that the entire state misses the detector, to disappear in or close to the beam pipe. Diffractive events have already been observed using this technique at ZEUS [11] and H1 [21].

Cases (2) and (3) correspond to replacing the lower pomeron-proton vertex in Fig. 11 by a diffractive excitation as in Fig. 2. The factor $\left|\beta_{B \mathbb{P}}(t)\right|^{2}$ in Eq. (画) is replaced by a factor from ordinary soft diffraction:

$$
\left|\beta_{B \mathbb{P}}(t)\right|^{2} \longrightarrow\left|\beta_{B \mathbb{P}}(t)\right|^{2}\left(1+\Delta_{B}(t)\right),
$$

where the correction factor $1+\Delta$ can obtained from the ratio of diffractive to elastic scattering:

$$
\frac{d \sigma^{\text {diff }}(A+B \rightarrow A+X)}{d t}=\frac{d \sigma^{\mathrm{el}}(A+B \rightarrow A+B)}{d t}\left(1+\Delta_{B}(t)\right)
$$

${ }^{4}$ When the pomeron's momentum fraction $x_{\mathbb{P}}$ is very small, the resolution of the measurement of the proton's momentum may not be sufficient to get a good determination of $x_{\mathbb{P}}$. In that case, measurement of the other detected particles will serve to determine $x_{\mathbb{P}}$ by the relation $x_{\mathbb{P}}=M_{X}^{2} / s$, a measurement which will be dominated by the particles close to the edge of the rapidity gap, and by particles with high transverse momentum - including the scattered electron in deep-inelastic events. The use of light-cone coordinates will facilitate this computation - see App. A. It should be emphasized that the scattered electron at HERA is often in a kinematic configuration that is completely analogous to the isolated proton in diffractive scattering, and that measurement of the rest of the final state has proved to be in practice an effective method to determine the quantity corresponding to $x_{\mathbb{P}}$. 
The cross section we use here for single diffraction has an integral over the mass of the diffractively excited system (the lower group of final-state hadrons in Fig. 2). Thus the correction factor $1+\Delta$ will depend on the limit that is placed on this system. In the case of the rapidity gap signature, this limit is given by requiring that the hadrons be within the beam-hole of the detector.

The use of (20) assumes the validity of Regge factorization in its simplest form for ordinary diffraction, elastic scattering and for hard diffractive scattering. Thus it predicts that the ratio of contributions from quasi-elastic scattering and diffractive excitation in rapidity gap events should be the same in DIS events as it is in low $p_{T}$ scattering with pure quasi-elastic scattering of the oppositely-directed beam particle. We can therefore use the CDF Roman Pot data on single diffraction [22] to estimate what goes down the beam pipe in events with a gap trigger. Using CDF's parameterization of their data, we estimate the cross section for $\bar{p}+p \rightarrow \bar{p}+p^{*}$ in which the $p^{*}$ has such a low invariant mass $(\lesssim 6 \mathrm{GeV})$ that it would completely miss a detector confined to $|\eta|<4$, to be approximately $3 \mathrm{mb}$. Meanwhile the integrated elastic cross section $\bar{p}+p \rightarrow \bar{p}+p$ is approximately $20 \mathrm{mb}$, so a rapidity gap trigger would correspond $87 \%$ of the time to a single $p$ going down the beam pipe.

The data on inelastic diffraction are actually somewhat inconsistent with regard to normalization [23]. This partly reflects differences in the kinematic ranges included by different experiments. More detailed analysis, with more data from modern colliders, is needed. Analysis [24] of diffractive data also does not give a pomeron trajectory identical to that obtained from data on total and elastic cross sections, so there may be significant contributions from multi-pomeron exchange.

It will be important to compare the rapidity gap trigger with the pure quasi-elastic diffractive trigger, using the ZEUS Roman Pots. This will provide a "renormalization factor" that can be used to relate the two types of trigger in other experiments where only the rapidity gap method is available. The observed value of this factor, and the possibility that it may vary with the deep inelastic kinematic variables, or be different for a low $p_{T}$ process such as quasi-elastic $\rho^{0}$ or $J / \psi$ production, will provide an interesting test of Regge factorization for the pomeron.

Meanwhile, if Regge factorization is at least close to the truth, the "renormalization factor" will be somewhat less than unity, corresponding to small contributions from the inelastic $p^{*}$ states, so it should be possible to estimate the quasi-elastic diffractive cross sections rather accurately from data based on the rapidity-gap method.

Whereas the measurement of a single diffracted hadron in the beam pipe allows one to measure a diffractive cross section that is differential in $t$, the rapidity gap signature allows only an integral over $t$ to be measured. This is sufficient for a measurement of parton densities in the pomeron, although it does not give information on the interesting subject of the $t$-dependence. It is convenient in this regard that the relevant detectors (CDF, D0, H1, and ZEUS) have very similar kinematic coverage relative to the proton beam pipe: Parton densities measured by the gap method, which implicitly contain a weighted average over $t$, will be directly comparable, with small corrections.

Significant Regge phenomenology would be required, however, to relate these measurements to those of UA8, who have a true quasi-elastic requirement with $-t$ in the range of 1 to $2 \mathrm{GeV}^{2}$. Normal soft-pomeron phenomenology cannot necessarily be applied at such 
large $|t|$. For example, we observe that there is a substantial change in the behavior of the $p \bar{p}$ elastic cross section below and above $-t \approx 1 \mathrm{GeV}^{2}[25$. Ordinary pomeron phenomenology works well only below this boundary. Above it, a Regge analysis is still appropriate $\left(p_{T} \ll \sqrt{s}\right)$; but possibly the pomeron becomes more like the perturbative pomeron of BFKL 26], than the conventional phenomenological soft pomeron.

There is no fundamental requirement that the parton densities in the pomeron must be independent of $t$. But the strongest $t$ dependence is presumably in $\beta_{B \mathbb{P}}(t)$, since that represents an elastic coupling. Any experimental information on the $t$-dependence would be welcome. If It is also possible that the amount of the breakdown of factorization, if it occurs, is $t$-dependent.

\section{NON-FACTORIZATION}

\section{A. Delta-function terms}

CFS [3] predict that there are non-factorizing leading twist terms in hard diffractive scattering, but only in certain processes. These terms behave kinematically as if the pomeron had a point-like component, like a photon. For example there should be such a term in diffractive jet production in hadron-hadron collisions, where it will appear as a peak in the cross section at $x_{b} / x_{\mathbb{P}} \approx 1$. In the simplest approximation to the theory the term is a deltafunction, proportional to $\delta\left(x_{b} / x_{\mathbb{P}}-1\right)$. However, the strength of the delta-function is not universal between different processes.

A simple way to look for the CFS mechanism is to reconstruct the values of the parton momentum fractions $x_{a}$ and $x_{b} / x_{\mathbb{P}}$ from the jet momenta in each event. On an event-by-event basis, the kinematic analysis should be done relative to the pomeron-proton center-of-mass frame, or by the use of light-cone coordinates. Then one would plot the cross section as a function of $x_{b} / x_{\mathbb{P}}$. If the CFS mechanism is valid, there should be a contribution peaked at $x_{b} / x_{\mathbb{P}} \approx 1$.

A full analysis must recognize that the jets will be smeared out, compared to the situation at the parton level. Thus, as in the UA8 analysis [6], either substantial unfolding is necessary, or the data and theory should only be compared after smearing the theory. Smearing by hadronization and detector resolution is much more noticeable for a delta-function than for a smooth function! Moreover, there will be a correction due to higher-order perturbative QCD corrections to the jet production, including production of 3 or more jets. A full analysis could best be done by proceeding from assumed parton densities, and including resolution, hadronization, higher order QCD, etc., to calculate a cross section to be compared with the data. One would then adjust the parton densities to fit the data. This analysis will of course have limited power to distinguish a genuine delta-function from a narrow peak, since the physical cross section will not display a true delta-function.

\footnotetext{
${ }^{5}$ Compare in particular the recent work by Sotiropoulos and Sterman [27] concerning the $t$ dependence of elastic scattering.

${ }^{6}$ In fact, when perturbative QCD predicts a cross-section to have a delta-function, or some other
} 


\section{B. The $\delta$ function is not exact}

It is only in the simplest approximation that the CFS coherent term is proportional to a delta-function at $x_{b}=x_{\mathbb{P}}$. Higher order corrections are likely to smear it into something that is merely strongly peaked at $x_{b} \approx x_{\mathbb{P}}$. Experimental resolution and jet hadronization will smear it even more. The real experimental signatures are that diffractive jet production should be much larger as $x_{b} \rightarrow x_{\mathbb{P}}$ than can be explained by anything but a density of partons in the pomeron that rises strongly in that region. CFS [3] predict that any similar excess in DIS is higher twist, i.e., that in DIS the fraction of the coherent (or super-hard) events falls off as a power of $Q$ as $Q$ increases.

The meaning of a delta-function in any calculation of a hard process is not that a physical cross section has the delta-function, but that the experimental and calculated cross sections should agree, up to higher-twist terms, only after they are both averaged with a smooth test function. That is, the theoretical prediction should be interpreted in the sense of distributions. (Compare Ref. [33].) In our case that means that the actual prediction is simply that there is a substantial excess in a narrow range of $x_{b} / x_{\mathbb{P}}$ close to 1 .

\section{Other non-factorization}

The CFS mechanism is a particularly clear example of the failure of the factorization theorem. In general, to prove [28 30] the factorization theorem for a large-momentumtransfer process in hadron-hadron scattering, one must require that the cross section be inclusive. This requirement is violated by a diffractive condition on the final state. Besides the CFS term, there can be a general failure of the factorization theorem which would manifest itself in a failure of the universality of the parton densities: a simultaneous fit of parton densities to many processes would fail.

The requirements of the final-state cancellation are much less stringent in deep inelastic scattering - see [3, 30]. So it is quite possible that the factorization theorem holds within that class of processes - more theoretical investigations are needed on this point. This restricted factorization theorem would be manifested by a universality of the parton densities within this subset of processes. Such processes include the diffractive components of $F_{1}$ and $F_{2}$ at different values of $Q$, and the production of various numbers of jets in DIS. (In jet production in DIS, one should require the jet(s) to have transverse momentum of order $Q$, rather than much larger than $Q$. Otherwise one can get something more like photoproduction, where factorization should fail, since photoproduction is just a special case of hadron-hadron scattering for the purposes of the factorization theorem.)

singular behavior, the prediction should be interpreted in the sense of the theory of distributions - see Sect. VIB. 


\section{Process dependence}

The coherent term predicted by CFS is a contribution not present in the IngelmanSchlein model, and is predicted to be quite different in different processes. Thus data for a suitable set of processes should not be consistently fit by a single set of parton densities, at least for the delta-function term. It is also not necessarily true that the continuum term in the parton densities is universal, or that the $t$-dependence of the delta-function term is similar to the continuum (Ingelman-Schlein) term.

To understand the process-dependence, one must understand CFS's reasoning. They model the pomeron by two-gluon exchange as in Fig. 3. One of the gluons (the 'hard gluon') carries most of the longitudinal momentum of the pomeron and the other gluon is relatively soft, at least in terms of its longitudinal momentum. The soft gluon is to be regarded as exchanged between two oppositely moving gluons (or a gluon and a quark), and one of these gluons is the 'hard gluon' in the pomeron. Since a diffractive requirement has been imposed on the final state, the usual cancellation [28 30] of the effects of soft gluons, which is responsible for QCD factorization in inclusive processes, no longer occurs.

Now a gluon only couples to color fields, so the corresponding graphs do not exist in DIS, where an incoming electron plays the role of the uppermost gluon in Fig. 3. Instead, in DIS, one must consider graphs like Fig. 世, as has been done by Donnachie and Landshoff [31] and by Nikolaev and Zakharov 32. In order for the kinematics to correspond to the coherent pomeron term, we assume that the quark and anti-quark coming from the virtual photon have transverse momenta comparable to the virtuality $Q$ of the photon. The quark and anti-quark thus give rise to jets to be associated with the hard scattering. Graphs like Fig. 4 exist and give an approximate delta-function, just like the graphs in diffractive hadroproduction. But since the second gluon of the pomeron attaches directly to the hard scattering, or to an out-going parton, the result is higher twist [3]. That is, if one analyzes the cross section in terms of a continuum plus a delta-function term, then the delta-function falls off as a power of $Q$ relative to the continuum. This is in contrast to the delta-function term in the hadroproduction of jets, which is predicted by CFS to scale. CFS note that the cross section calculated by Donnachie and Landshoff [31] agrees with this observation.

\section{E. Systematics}

We summarize here the systematics of the predicted delta-function terms:

1. In DIS and the direct photoproduction of jets, the delta-function term should be higher twist, i.e., it should die away as a power of $Q$ or of jet transverse energy $E_{T}$ when one tries to make a scaling test.

2. In hadroproduction of jets, the delta-function should be of leading twist: it will scale and should not fall off as a power of increasing jet $E_{T}$.

3. In $W$ and $Z$ production, there should be at most a small delta-function term when $q_{T}$, the transverse momentum of the $W / Z$, is small. But as one increases $q_{T}$, a jet will 
appear. If one analyzes the process in terms of the kinematics of the $W / Z$ plus jet(s) system, there should be a delta-function term which will scale.]

4. Resolved jet production in diffractive photoproduction should also have a leading-twist delta-function, at least if there is sufficient kinematic range to allow the process.

How to determine experimentally whether some diffractive quantity is leading or higher twist is explained in Sect. VII.

In addition, there may be substantial $t$-dependence. The most reliable prediction of the coherent delta-function term is at sufficiently large $|t|$ that the perturbative model is at least qualitatively valid. At low $|t|$ - an unambiguously non-perturbative region for the pomeron - one cannot trust the two-gluon exchange model.

When a rapidity gap signature is used, there is an integral over the unmeasured value of the pomeron's momentum transfer. This integral is dominated by very low $|t|$ - certainly well below $1 \mathrm{GeV}^{2}$. Hence one cannot necessarily expect perfect consistency between the jet production reported by UA8 [6] and the jet production that would be measured by CDF and D0 using a rapidity gap signature for pomeron exchange. The difference itself is an important probe of the space-time structure of the pomeron. Of course, a test of the CFS mechanism is that there should be consistency between the excess of events at large $x_{b} / x_{\mathbb{P}}$ measured by UA8, and a similar excess that could be measured by CDF if Roman Pot detectors are reinstalled and used in a kinematic region corresponding to that of UA8.

\section{CONSISTENCY CHECKS: SCALING TESTS}

\section{A. Pomeron exchange}

To check that a measurement of a hard diffractive process is really associated with pomeron exchange, one must verify the $x_{\mathbb{P}}$ dependence. We are in effect defining the pomeron as whatever is responsible for the leading power behavior as $x_{\mathbb{P}} \rightarrow 0$. For the name 'pomeron' to be appropriate, the intercept, $\alpha_{\mathbb{P}}(0)$, must be close to (or above) unity, the approximate value that appears in soft processes like the total cross section for hadron-hadron scattering.

Diffractive cross sections go like $d \sigma / d x_{\mathbb{P}} \propto x_{\mathbb{P}}^{1-2 \alpha_{\mathbb{P}}}$ as $x_{\mathbb{P}} \rightarrow 0$, with fixed $x_{\mathbb{P}} s$ and $t$. (Thus we are taking $s \rightarrow \infty$.) This power law is roughly $x_{\mathbb{P}}^{-1}$ since $\alpha_{\mathbb{P}} \approx 1$. However, if one makes a jet measurement at a given $\sqrt{s}$ and $E_{T}$, the cross sections in Eq. (雨) etc., also have $x_{\mathbb{P}}$-dependence from the factor $f_{b / \mathbb{P}}\left(x_{b} / x_{\mathbb{P}}\right)$ of the parton density in the pomeron. As $x_{\mathbb{P}}$ is decreased, $x_{b} / x_{\mathbb{P}}$ increases, and the generally strong zero in a parton density for $x \rightarrow 1$ greatly reduces the cross section. Thus, even for pure pomeron exchange, one should not expect to see $x_{\mathbb{P}}^{-1}$ behavior in the actual hard diffractive scattering cross section at fixed $\sqrt{s}$. The most reliable way to look for the $x_{\mathbb{P}}^{1-2 \alpha_{\mathbb{P}}}$ behavior is by a scaling test: increase $s$ and decrease $x_{\mathbb{P}}$ while keeping fixed $x_{\mathbb{P}} s, t$ and the definition of the hard scattering (e.g., the

7 The reason for needing an extra jet in $W / Z$ production to get the delta-function term is that the hard process without the jet is $\mathbb{P}+q \rightarrow W / Z$. The only way to balance the baryon number is to emit a soft quark into the final state, which will be suppressed. 
value of $E_{T}$ ). When one uses a rapidity gap signature, the condition of fixing $t$ is replaced by an integral over $t$.

In the absence of a scaling test, one must analyze the data at each value of $x_{\mathbb{P}}$, using Eq. (4) to obtain parton densities. The parton densities should then be consistent between different values of $x_{\mathbb{P}}$. If not, there is some contamination by exchange of Reggeons other than the pomeron. Alternatively, one could analyze the $x_{\mathbb{P}}$ dependence of the cross section after factoring out an assumed or fitted factor for the cross section for pomeron-induced hard scattering. That factor could be, for example, everything to the right of $x_{\mathbb{P}}^{1-2 \alpha_{\mathbb{P}}}$ in Eq. (四).

To see the pomeron clearly, one should do this analysis over a range of $x_{\mathbb{P}}$ that includes the transition between Reggeon and pomeron regions. An appropriate range might be $0.85<1-x_{\mathbb{P}}<0.98$. The range should include a definitely non-pomeron region, as well as the region nearest to $x_{\mathbb{P}}=0$ that one expects to be pomeron-dominated.

The scaling test above ("Regge scaling") is easier to do for diffractive deep inelastic scattering. The diffractive structure functions are given by Eqs. (13) and (14), and the Regge scaling test is to decrease $x_{b j}$ and $x_{\mathbb{P}}$ while holding $Q^{2}$ and $x_{b j} / x_{\mathbb{P}}$ fixed. This test can be done at fixed lepton-hadron center-of-mass energy, even though the ideal test is to vary $s$ as well, since that avoids all questions about the ratio $F_{1} / F_{2}$. (Then one can keep $y$ fixed, as well as $Q^{2}$ and $x_{b j} / x_{\mathbb{P}}$.)

One should also analyze the $t$ dependence. For that purpose, parton densities in the pomeron are to be regarded as purely non-perturbative unknown quantities. As $|t|$ increases, it is natural that the pomeron should become a smaller object, in analogy with the way a virtual photon becomes more point-like as $Q$ increases. Therefore the parton densities could change very noticeably with $t$. Depending on the design of the Roman Pots, the measurements may go out to relatively large $|t|$ by the standards of soft hadron-physics. If so, the $t$ dependence of $\alpha_{\mathbb{P}}$ - see Eq. (10) — will have to be measured when assessing the $x_{\mathbb{P}}$-dependence.

Notice that the UA8 [6] experiment was sensitive to $-t$ between 1 and $2 \mathrm{GeV}^{2}$, a range that is beyond the conventional range of fits to diffractive physics. It is therefore important to measure the pomeron-hadron coupling $\beta_{p \mathbb{P}}(t)$ at these values of $t$. That is, a program of new measurements of conventional diffractive and elastic physics is essential for the full implementation of our program. With the large $\sqrt{s}$ available at the colliders, the kinematic region can go well beyond that available when diffractive physics was a common subject of experimental investigation. In particular, one can go to substantially larger $-t$, where perturbative methods for the pomeron may start to be applicable. This would be in the range up to a few $\mathrm{GeV}^{2}$. Along with measurements of single diffractive excitation, the program should include measurements of elastic scattering, where there has been interesting recent theoretical work, for example, by Sotiropoulos and Sterman [27].

As to the rapidity-gap method, the Regge predictions for making rapidity gaps in soft scattering need to be tested, since the analysis of the hard scattering depends on the applicability of standard Regge theory to low transverse momentum phenomena.

With elastic diffraction, where the diffracted proton is detected (in a Roman Pot detector), a further test of the Regge behavior can be made by also measuring the cross section when the diffracted proton is replaced by a hadron or hadrons in a different charge state. This only works with a suitable detector, of course. The Regge exchange that gives the diffraction can no longer have vacuum quantum numbers, so that it cannot be a pomeron. 
In formulae like Eqs (4) and (7), $\alpha_{\mathbb{P}} \approx 1$ is replaced by a smaller value, for non-leading Reggeon exchange.

\section{B. Bjorken Scaling}

To verify that hard diffractive scattering is as we have described it, one must also verify that it obeys approximate Bjorken scaling. (Of course, QCD predicts logarithmic violations of Bjorken scaling for hard diffractive processes, just as for inclusive hard scattering. But the dominant issue here concerns the power law.)

In the case of deep inelastic scattering, a test of Bjorken scaling involves varying $Q^{2}$, while keeping $x_{b j} / x_{\mathbb{P}}, x_{\mathbb{P}}$ and $t$ fixed. The diffractive structure functions in Eqs. (13) and (14) should be approximately constant. Fixing $x_{b j} / x_{\mathbb{P}}$ while varying $Q^{2}$ is exactly the test of Bjorken scaling for deep inelastic scattering with the pomeron treated as the target. Fixing $x_{\mathbb{P}}$ and $t$ ensures that there are no confounding effects from the variation of the pomeron factors in Eqs. (13) and (14). When one uses a rapidity gap signature, the condition of fixing $t$ is again replaced by an integral over $t$.

For the other processes, one makes the obvious generalizations. For example, consider diffractive jet production in hadron-hadron collisions. Bjorken scaling involves increasing the jet $E_{T}$ while holding fixed the hard scattering scaling variables $x_{a}$ and $x_{b / \mathbb{P}}$, and the pomeron variables $x_{\mathbb{P}}$ and $t$. (Again, the condition of fixing $t$ may be replaced by integrating over it.) The variables $x_{a}$ and $x_{b} / x_{\mathbb{P}}$ are measured from two-jet production, by assuming parton-model kinematics in the pomeron-proton collision. The cross section in Eq. (四) will be proportional to $1 / E_{T}^{2}$. A cross section differential in $E_{T}, d \sigma / d t d x_{\mathbb{P}} d E_{T}$, would scale as $1 / E_{T}^{3}$. There will be the usual logarithmic violations of this scaling. This test necessarily involves varying the beam energy, or by bringing in more theory and using a fit of the diffractive parton densities.

\section{Combination}

One test that can be done at fixed $s$ is to increase $E_{T}^{2}$, while holding $x_{a}$ and $x_{b} / x_{\mathbb{P}}$ fixed. This scaling limit has $x_{\mathbb{P}} \propto E_{T}^{2}$. The cross-section $d \sigma / d t d x_{\mathbb{P}}$, integrated over a range of $E_{T}$, will scale like $1 / E_{T}^{4 \alpha_{\mathbb{P}}}$ from a combination of Regge and Bjorken scaling.

For DIS, the corresponding test has $Q^{2}$ being increased with $x_{b j} / x_{\mathbb{P}}$ fixed, and with $x_{\mathbb{P}}$, or equivalently $x_{b j}$, being increased in proportion to $Q^{2}$. The diffractive structure functions in Eqs. (13) and (14) should scale like

$$
\begin{aligned}
& \frac{d F_{1}^{\text {diffractive }}\left(x_{b j}, Q ; t, x_{\mathbb{P}}\right)}{d t d x_{\mathbb{P}}} \propto \frac{1}{Q^{4 \alpha_{\mathbb{P}}}}, \\
& \frac{d F_{2}^{\text {diffractive }}\left(x_{b j}, Q ; t, x_{\mathbb{P}}\right)}{d t d x_{\mathbb{P}}} \propto \frac{1}{Q^{4 \alpha_{\mathbb{P}}-2}},
\end{aligned}
$$

in this limit with $x_{\mathbb{P}} \propto Q^{2} / s$. The different powers of $Q$ for $F_{1}$ and $F_{2}$ reflect the different powers of $x_{\mathbb{P}}$ in Eqs. (13) and (14). 


\section{SUMMARY OF PROCESSES TO BE MEASURED}

\section{A. Hard Processes}

Here follows a list of the hard diffractive processes that need to be investigated:

1. $e p \rightarrow e X p$ : diffractive DIS, as a function of $x_{b j}, Q, x_{\mathbb{P}}$, and $t$.

2. The same with 2 or more jets of transverse momentum of order $Q$.

3. $\gamma p \rightarrow X+$ jets $+p$ : diffractive photoproduction of jets, as function of $p_{T \text { jet }}, x_{\mathbb{P}}$, and $t$; both direct and resolved processes.

4. $p \bar{p} \rightarrow p+$ jets $+X$ : diffractive hadroproduction of jets.

5. The same for $W, Z$ and high- $p_{T}$ production of prompt photons.

6. $\gamma+p \rightarrow \rho+$ jets $+X$ : diffractive photoproduction of jets, with the photon being diffracted to a $\rho$-meson.

7. The same with the $\rho$ replaced by any other vector meson, e.g., $\omega, \phi, J / \psi$.

8. All of the above with the diffracted hadron replaced by a system separated from the rest of the event by a rapidity gap.

9. Any of the above with the diffracted hadron replaced by a hadron in a different charge state, so that the process cannot occur by pomeron exchange. Such processes should be suppressed by a power of $1 / x_{\mathbb{P}}$ compared to processes where pomeron exchange is allowed.

\section{B. Soft Processes}

Here follows a list of soft diffractive processes that need to be further investigated in order to provide better quantitative information for the soft part of hard diffractive processes:

1. Elastic hadron-hadron scattering is already well-known as a function of $s$ and $t$.

2. $p \bar{p} \rightarrow p \bar{p}^{*}$ : Single diffraction, as a function of $s, t$, and $x_{\mathbb{P}}$. Compare with the tripleRegge formalism.

3. $p \bar{p} \rightarrow p^{*} \bar{p}^{*}$ : Double diffraction, with $p^{*}, \bar{p}^{*}$ being systems of relatively low mass which will be separated by a rapidity gap. Compare with the triple-Regge formalism.

4. All of the above, with the $\bar{p}$ replaced by a photon $\gamma$, and the diffracted $\bar{p}$ replaced by a vector meson $(\rho, \omega, \phi, J / \psi)$. One can also do this for a large virtuality photon and for large $t$ - see Ref. [20].

5. Any of the above with the diffracted hadron replaced by a hadron in a different charge state, so that the process cannot occur by pomeron exchange. Such processes should be suppressed by a power of $1 / x_{\mathbb{P}}$ compared to processes where pomeron exchange is allowed. 


\section{CONCLUSIONS}

We have proposed a systematic investigation of hard diffractive scattering, with the unifying element being a measurement of the parton densities in the pomeron, as defined by the Ingelman-Schlein model. Since there is a prediction that this model should fail, the results of the measurements must have non-trivial implications for our understanding of non-perturbative QCD. (Even though the Ingelman-Schlein model may fail, the UA8 data show order-of-magnitude agreement with it when a reasonable ansatz is used for the parton densities. Thus the model is close enough to remain a useful basis for planning experiments.)

By coordinating measurements between HERA and Fermilab, one can readily measure the flavor-separated quark and gluon densities, and then perform non-trivial tests of QCD factorization. Since the distributions of $u, d, \bar{u}$ and $\bar{d}$ quarks in the pomeron are equal, two processes suffice to make the measurement - say DIS and direct photoproduction of jets. Then other hard-scattering processes, e.g. hadroproduction of jets or of $W$ or $Z$ bosons, with a diffractive requirement imposed, provide tests of the picture.

In view of the possibility that factorization might hold for deep inelastic processes, while failing for other processes, one should perhaps attempt to treat diffractive DIS without and with the measurement of final-state jets as the basic processes to measure the parton densities in the pomeron. Since the diffractive requirement will reduce the effective centerof-mass energy, the transverse momenta of the jets may be so low as to make jet physics marginal. But it is worth trying this. There should be enough consistency requirements (e.g., in the $Q$ dependence) to give a test of factorization within the DIS processes alone.

The diffractive requirement can be imposed by Roman Pot ("quasi-elastic", "exclusive diffractive" condition) or by a simple rapidity gap requirement. The similar rapidity coverage of the four experiments CDF, D0, H1 and ZEUS implies that rapidity gap measurements should be rather directly comparable between the different experiments. The data from UA8 on diffractive jet production and from the other experiments with a rapidity gap condition indicates that there will be plenty of data, with rates on the order of $1 \%$ or higher of the event rate without the diffractive condition on the final state.

The question of whether the pomeron is dominated by gluons or by quarks is an important issue that needs to be resolved by experiment, as is the issue of whether or not the momentum sum rule is valid. The measurements we propose will answer these questions.

The $t$-dependence of the parton densities in the pomeron is also of considerable interest. It can only be probed using the Roman Pot technique.

CFS [3] predict that the Ingelman-Schlein picture will break down, in that there will be, in certain processes, an excess of events at $x_{b} / x_{\mathbb{P}} \approx 1$. These should exhibit Bjorken scaling when the characteristic momentum of the hard scattering is increased. The excess should be present in hadroproduction of jets, and appears to have been observed by UA8 [6]. In a process such as deep inelastic scattering or in the "direct" (as opposed to "resolved") component of jet photoproduction, the excess, if any, is predicted to be of higher twist, i.e., suppressed by a power of $1 / Q\left(1 / p_{T}\right.$, in the case of photoproduction). The systematics of a

breakdown of factorization in diffractive processes, as a function of the process and of the kinematic variables, in particular $t$, will provide important information on the space-time structure of the pomeron.

Since part of the formulae for the cross sections involve conventional Regge theory, it 
is important to test Regge theory further at the energies now available, particularly with regard to the rapidity gap cross sections [23,34.

Another possible test of the Regge factorization could be made using two-jet production in double pomeron exchange (DPE) interactions at the Tevatron. This could be done using the rapidity-gap method. In the case of D0, signals in their forward and backward scintillation counters have been required as a part of all triggers, thereby preventing triggering on DPE event. Those counters, and similar ones in CDF, could in fact be used in veto mode to trigger on DPE events [35]. The CFS argument should also apply to jet production in DPE.

\section{ACKNOWLEDGMENTS}

This work was supported in part by the U.S. Department of Energy under grant DEFG02-90ER-40577, by the U.S. National Science Foundation, and by the Texas National Laboratory Research Commission grant to the CTEQ collaboration. One of the authors (JJW) wishes to acknowledge partial support from the DESY Directorate in 1993.94. We would like to thank many colleagues for discussions, notably our colleagues in the CTEQ collaboration, and L. Frankfurt, E.M. Levin, and M. Strikman. We also wish to thank the anonymous referee for a number of thoughtful comments that have improved the content and clarity of the paper.

\section{APPENDIX A: LIGHT-CONE COORDINATES}

In this appendix, we define light-cone coordinates. Then we show how they can be used to make measurements of, for example, the momentum of an exchanged pomeron from the hadronic final-state without observation of the diffracted proton.

Consider the reaction shown in Fig. 5 where incoming particle $p_{2}$ (traveling in the $+z$ direction) emits a pomeron $\mathbb{P}$ which then interacts with particle $p_{1}$ (traveling in the $-z$ direction). The diffracted particle $p_{2}^{\prime}$ emerges containing a high fraction $x(\gtrsim 0.95)$ of the momentum of particle $p_{2}$. The pomeron carries a momentum fraction $x_{\mathbb{P}}=1-x$. The direction of the $+z$ axis is the same as in the ZEUS detector when particle $p_{2}$ is the proton.

One way of determining the momentum fraction of the pomeron ( $\grave{a}$ la UA8) is to measure the momentum of the diffracted particle $p_{2}^{\prime}$, using Roman Pot detectors. Currently, none of the collider detectors at the Tevatron are equipped with Roman Pot detectors. But this should be considered as an upgrade option. The ZEUS experiment recorded some data in 1993 with some partially equipped Roman Pot detectors.

It is also of interest to measure the cross section when the diffracted particle is replaced by a system of relatively low mass.

Another determination of $x_{\mathbb{P}}$ can be carried out by measuring the momenta of the other particles $q_{1}, \ldots, q_{n}$ in the final-state. When these are expressed in light-cone coordinates, it is easy to show that there is a rapidity gap on the side of the detector towards which $p_{2}$ travels (the $+z$ direction). The computation of $x_{\mathbb{P}}$ is dominated by the particles close to the edge of the rapidity gap and by particles of high transverse momentum, as we will 
also show. The same method is already used to reconstruct the photon kinematics from the hadron final-state in electron-proton scattering [11,36].

The energy and $z$ component of the momentum of each of the outgoing particles $q_{i}$ can be written

$$
\begin{aligned}
& q_{i}^{0}=m_{i}^{T} \cosh y_{i}, \\
& q_{i}^{z}=m_{i}^{T} \sinh y_{i},
\end{aligned}
$$

where $m_{i}^{T}\left(=\sqrt{q_{i}^{T^{2}}+m_{i}^{2}}\right)$ is the transverse mass, and $y_{i}$ is the rapidity of the particle. We can define the following coordinates

$$
\begin{aligned}
& {q_{i}}^{+}=\frac{q_{i}^{0}+q_{i}^{z}}{\sqrt{2}}=m_{i}^{T} e^{y i} / \sqrt{2}, \\
& q_{i}^{-}=\frac{q_{i}^{0}-q_{i}^{z}}{\sqrt{2}}=m_{i}^{T} e^{-y_{i}} / \sqrt{2}, \\
& q_{i}^{T}=\left(q_{i}^{x}, q_{i}{ }^{y}\right)
\end{aligned}
$$

which are called light-cone coordinates. When the mass of a particle is small compared with its transverse momentum, the rapidity is well approximated by the pseudorapidity $\eta_{i}$, which is determined directly from the polar angle $\theta: \eta=-\ln \tan (\theta / 2)$. So it is useful to consider the $\eta$ - $\phi$ plot, which is shown in Fig. 6, for the hard diffractive production of 2 jets.

As above, $x$ is the fraction of the momentum taken by the outgoing particle $p_{2}^{\prime}$. This is most conveniently defined in term of light-cone coordinates:

$$
x=\frac{p_{2}^{\prime+}}{p_{2}^{+}} .
$$

Then momentum conservation, $p_{2}=p_{2}^{\prime}+\sum q_{i}-p_{1}$, yields

$$
\begin{aligned}
x_{\mathbb{P}} & \equiv \frac{p_{2}^{+}-p_{2}^{+}}{p_{2}^{+}} \\
& =\frac{\sum q_{i}^{+}-p_{1}^{+}}{p_{2}^{+}} .
\end{aligned}
$$

Since $p_{1}{ }^{+} / p_{2}{ }^{+}=\mathcal{O}\left(m^{2} / s\right)$, we can neglect $p_{1}{ }^{+}$, except if the event is almost exactly elastic, which is not the case when there is a hard scattering. Hence

$$
x_{\mathbb{P}} \approx \sum \frac{m_{i}^{T} e^{y_{i}}}{E_{2}+p_{2}^{z}} \approx \sum_{i} \frac{\sqrt{q_{i}^{T^{2}}+m_{i}^{2}} e^{-\left(y_{p_{2}}-y_{i}\right)}}{m_{p}} .
$$

For diffraction, we require $x_{\mathbb{P}}$ to be small. Then Eq. (A5) shows that the rapidities of all the particles $q_{i}$ must be substantially less than the rapidity of the incoming proton $p_{2}$. Hence we must have a rapidity gap, and to the extent that there are few particles of very low transverse momentum, this implies a gap in pseudorapidity, as we claimed earlier. It is important to have calorimetry coverage over as large a rapidity interval as possible in order to confirm the presence of this gap. 
Moreover, only those particles with the largest $q^{T}$ and/or largest rapidity contribute significantly to the sum. Substituting pseudorapidity $\left(\eta_{i}\right)$ for rapidity $\left(y_{i}\right)$ should result in a small error in the determination of $x_{\mathbb{P}}$. Hence, Eq. (A5) gives an effective method for the measurement of $x_{\mathbb{P}}$. Note that the lack of coverage in the detector on the side opposite to the diffracted hadron is irrelevant.

In a typical "minimum-bias" final state, there is an approximately uniform distribution of hadrons in $\eta$ and $\phi$ in the non-gap region. Hence $x_{\mathbb{P}}=\mathcal{O}\left(e^{-\eta_{\text {gap }}}\right)$, where $\eta_{\text {gap }}$ is the difference between the (pseudo-)rapidity of the edge of the gap and the rapidity of the proton. For example, if $\eta_{\text {gap }}=-3, x_{\mathbb{P}}$ is less than or equal to .05 .

\section{APPENDIX B: DIFFRACTIVE STRUCTURE FUNCTIONS}

The right-hand sides of (13) and (14) are, of course, written on the assumption that the Ingelman-Schlein model is valid. Within the model, structure functions in the pomeron are defined in exactly the same way as structure functions in the proton.

One of our intentions is to test the Ingelman-Schlein model. So the diffractive structure functions $F_{i}^{\text {diffractive }}$ on the left-hand sides of these equations need to be defined. That is the purpose of this appendix.

A diffractive DIS process is just semi-inclusive DIS, with the final state restricted to a particular kinematic region for the detected outgoing hadron. So there are actually five structure functions [37], only four of which contribute to the unpolarized cross section. What we will show is that the cross section is almost certainly dominated by two of these, which in Kingsley's [38] conventions are like the usual $F_{1}$ and $F_{2}$. Kingsley's conventions are more convenient for our purposes than those of Meng et al. [39. We write

$$
\begin{aligned}
W^{\mu \nu}= & \frac{1}{4 \pi} \sum_{X} \int\left\langle P\left|J^{\nu}(0)\right| P^{\prime}, X\right\rangle\left\langle p^{\prime},\left.X\right|^{J}(0) \mid P\right\rangle \\
= & \left(-g^{\mu \nu}+\frac{q^{\mu} q^{\nu}}{q^{2}}\right) V_{1}+\frac{P^{\mu} P^{\nu}}{p \cdot q} V_{2} \\
& +\frac{P_{T}^{\prime \mu} P_{T}^{\prime \nu}}{p \cdot q} V_{3}+\frac{P_{T}^{\prime \mu} P^{\nu}+P^{\mu} P_{T}^{\prime \nu}}{2 p \cdot q} V_{4}+i \frac{P_{T}^{\prime \mu} P^{\nu}-P^{\mu} P_{T}^{\prime \nu}}{2 p \cdot q} V_{5},
\end{aligned}
$$

where $P^{\mu}=p^{\mu}-p \cdot q / q^{2}$, and $P_{T}^{\prime \mu}$ is component of the momentum of the outgoing diffracted hadron that is transverse to both $p$ and $q$. In the diffractive limit, $P_{T}^{\prime \mu}$ is the component of the momentum of the outgoing hadron that is transverse to the collision axis. We have written the scalar structure functions in terms of dimensionless structure functions. The first two structure functions have exactly the same form as for inclusive deep inelastic scattering. We have used symbols $V_{i}$ to avoid confusion with the usual structure functions $F_{i}$. The fifth structure gives a zero contribution when contracted with the symmetric leptonic tensor in unpolarized scattering.

Normal power counting for the hard scattering shows that each of the $V_{i}$ scales in the Bjorken limit. This power counting applies before the more detailed arguments needed to get factorization are applied; it is really dimensional counting of powers of the large mass scale in the problem. Since there is a factor of a small transverse momentum with the $V_{3}$ 
and $V_{4}$ structure functions, they give a nonleading power when contracted with the leptonic tensor. So to a good approximation, only $V_{1}$ and $V_{2}$ contribute.

When we contract with the leptonic tensor, the dependence on the variables $x_{b j}$ and $y \equiv q \cdot p / l \cdot p$ is the same as in inclusive deep inelastic scattering. The cross section also depends on the variables $t$ and $x_{\mathbb{P}}$ of the diffracted hadron. By multiplying $V_{1}$ and $V_{2}$ by a factor we may therefore write the cross section as

$$
\frac{d \sigma}{d x d y d t d_{\mathbb{P}}}=\frac{4 \pi \alpha^{2} s}{Q^{4}}\left[x y^{2} \frac{d F_{1}^{\text {diffractive }}}{d t d_{\mathbb{P}}}(1-y) \frac{d F_{2}^{\text {diffractive }}}{d t d_{\mathbb{P}}}\right] .
$$

This defines the diffractive structure functions in Eqs. (13) and (14) in terms of the $y$ dependence of the cross section. Note that although we use the label "diffractive" on these structure functions, we do not mean to imply that they are due to diffraction (i.e., pomeron exchange). Rather the label only indicates that they are for a process and kinematic configuration that is appropriate for investigating diffraction.

\section{APPENDIX C: NORMALIZATION}

In this section we provide some arguments about the normalization of Eq. (雨). We are considering inclusive cross sections for the process $A+B \rightarrow X+B^{\prime}$. Elementary manipulations show that the cross section differential in the variables for $B^{\prime}$ is

$$
\frac{d \sigma\left(A+B \rightarrow X+B^{\prime}\right)}{d t d x_{\mathbb{P}}}=\frac{1}{32 \pi^{2} s} \frac{1}{i} \operatorname{disc} \mathcal{M},
$$

where $\operatorname{disc} \mathcal{M}$ is the appropriate discontinuity of the amplitude shown in Fig. 7. We have made approximations valid for $x_{\mathbb{P}} \ll 1$ and $s \gg m^{2}$.

To get a jet cross section, as in Eq. (团), one would restrict the integral over the final state $X$. But suppose first that one integrates over all $X$, to get the normal single diffractive cross section. Moreover, let us take the triple Regge limit: $m^{2} \ll M_{X}^{2} \ll s$ with $t$ fixed. (Note that $x_{\mathbb{P}}=M_{X}^{2} / s$.) Then the triple Regge formula for the cross section is

$$
\frac{d \sigma\left(A+B \rightarrow X+B^{\prime}\right)}{d t d x_{\mathbb{P}}}=\frac{1}{16 \pi}\left|\beta_{B \mathbb{P}}(t)\right|^{2}\left|\xi\left(\alpha_{\mathbb{P}}(t)\right)\right|^{2} x_{\mathbb{P}}^{1-2 \alpha_{\mathbb{P}}(t)} G_{\mathbb{P P P}}(t) \beta_{A \mathbb{P}}(0) M_{X}^{2 \alpha_{\mathbb{P}}(0)-2}
$$

where we have used the normalizations of Ref. [18]. The signature factor

$$
\xi\left(\alpha_{\mathbb{P}}\right)=-\frac{\tau+e^{-i \pi \alpha_{\mathbb{P}}}}{\sin \pi \alpha_{\mathbb{P}}},
$$

is close to $i$ for the pomeron, which has even signature $\tau=1$.

To get a Pomeron-proton cross-section, one naturally would factor out the factors associated with the $B \mathbb{P} B^{\prime}$ vertex. According to 18 the result is

$$
\sigma_{\text {tot }}(A \mathbb{P})=G_{\mathbb{P P P}}(t) \beta_{A \mathbb{P}}(0) M_{X}^{2 \alpha_{\mathbb{P}}(0)-2}
$$

which is exactly the formula one would use for the total cross section for hadron-hadron scattering, with the triple-pomeron coupling $G_{\mathbb{P P P}}(t)$ replacing a pomeron-hadron coupling. The formulae given by Kaidalov [19] are completely consistent with the above. 
Now, in both [18] and [19], Eq. (C2) and Eq. (C4) are claimed to be valid when the pomeron exchanged with the $B$ particle is replaced by any other Regge trajectory, as would be appropriate for a charge exchange process, for example.

One could argue that the physical region for diffraction is a long way from any particle pole on the pomeron trajectory, and thus that it is quite ambiguous as to how to factor off the pomerons to go from Eq. (C2) to Eq. (C4). But suppose one has a theory with a massless scalar particle $R$. Then one can construct the same diffractive process as in Eq. (C2), but with quantum numbers for $B$ and $B^{\prime}$ such that the leading term in the cross section has $R$ exchange instead of pomeron exchange. Then Eq. (C2) is replaced by

$$
\frac{d \sigma\left(A+B \rightarrow X+B^{\prime}\right)}{d t d x_{\mathbb{P}}}=\frac{1}{16 \pi}\left|\beta_{B R B^{\prime}}(t)\right|^{2}\left|\xi\left(\alpha_{R}(t)\right)\right|^{2} x_{\mathbb{P}}^{1-2 \alpha_{R}(t)} G_{R R \mathbb{P}}(t) \beta_{A \mathbb{P}}(0) M_{X}^{2 \alpha_{\mathbb{P}}(0)-2} .
$$

The Regge pole for $R$ will be of even signature, and close to $t=0$ we can write

$$
\alpha_{R}(t)=\alpha_{R}^{\prime} t
$$

The signature factor will therefore have a pole at $t=0$ :

$$
\xi\left(\alpha_{R}(t)\right) \rightarrow \frac{-2}{\pi \alpha_{R}^{\prime} t} \text { as } t \rightarrow 0 .
$$

This pole is at the edge of the physical region for diffraction, so we may use the LSZ reduction method to obtain the cross section for $A R$ scattering. First we obtain the discontinuity in Eq. (C1) from Eq. (C5). Then for each of the two $R$ exchanges, we have to remove the factor $1 / t$ and the square root of the residue in $B B^{\prime}$ elastic scattering:

$$
\mathcal{M}_{B B^{\prime} \rightarrow B B^{\prime}}=\beta_{B R B^{\prime}}(t)^{2} \xi\left(\alpha_{R}(t)\right) .
$$

The result is that the discontinuity at $t=0$ of the $A R$ elastic scattering amplitude is

$$
\operatorname{disc} \mathcal{M}(t=0, A R \rightarrow A R)=\frac{4 i}{\alpha_{R}^{\prime}} s^{\alpha_{\mathbb{P}}(0)} G_{R R \mathbb{P}}(0) \beta_{A \mathbb{P}}(0),
$$

where $M_{X}^{2}$ in Eq. (C5) is now replaced by $s$. The corresponding total cross section is

$$
\sigma_{\text {tot }}(A R)=\frac{2}{\alpha_{R}^{\prime}} s^{\alpha_{\mathbb{P}}(0)-1} G_{R R \mathbb{P}}(0) \beta_{A \mathbb{P}}(0) .
$$

This is in contradiction with Eq. (C4), which is supposed to be valid generally, and not just for the pomeron-proton cross section. 


\section{REFERENCES}

[1] P.D.B. Collins, "An Introduction to Regge Theory and High Energy physics" (Cambridge University Press, 1977); A. Donnachie and P.V. Landshoff, Phys. Lett. B296, 227 (1992); K. Goulianos, Phys. Rep. 101, 169 (1983); references therein.

[2] G. Ingelman and P. Schlein, Phys. Lett. 152B, 256 (1985).

[3] J.C. Collins, L. Frankfurt and M. Strikman Phys. Lett. B307, 161 (1993).

[4] L. Frankfurt and M. Strikman, Phys. Rev. Lett. 64, 1914 (1989).

[5] L. Frankfurt, "Hard Diffraction Processes at Colliders", talk at F.A.D. Meeting at Dallas, TX, 14 March 1992.

[6] UA8 Collaboration, (A. Brandt et al.), Phys. Lett. B297, 417 (1992).

[7] E.M. Levin, private communication.

[8] A. Berera and D.E. Soper, Phys. Rev. D50, 4328 (1994).

[9] G. Ingelman, "Partons and QCD effects in the pomeron", preprint DESY 93-109.

[10] J. Bartels and G. Ingelman, Phys. Lett. B235, 175 (1990). A. Donnachie and P.V. Landshoff, Phys. Lett. 191B, 309 (1987), erratum: ibid. 198B, 590 (1987); P. Bruni and G. Ingelman, "Diffractive hard scattering at $e p$ and $p \bar{p}$ colliders", preprint DESY93-187, Presented at Europhysics Conference on High Energy Physics, Marseille, 22-28 Jul. 1993; H.-J. Lu and J. Milana, Phys. Lett. B313, 234 (1993); N.N. Nikolaev and B.G. Zakharov, "The triple pomeron regime and the structure function of the pomeron in the diffractive deep inelastic scattering at very small $x$ ", preprint LANDAU-16-93, hep-ph/9306230; P. Bruni and G. Ingelman, Phys. Lett. B311, 317 (1993); G. Ingelman and K. Prytz, Z. Phys. C58, 285 (1993); E. Levin and M. Wusthoff, Phys. Rev. D50, 4306 (1994); G. Ingelman and K. Janson-Prytz, Phys. Lett. B281, 325 (1992); N. Nikolaev and B. G. Zakharov, Z. Phys. C53, 331 (1992); N. Arteaga-Romero et al., Mod. Phys. Lett. A4, 645 (1989).

[11] ZEUS Collaboration (M. Derrick et al.), Phys. Lett. B315, 481 (1993); and "Observation of jet production in deep inelastic scattering with a large rapidity gap at HERA", DESY preprint 94-063.

[12] D0 Collaboration (S. Abachi et al.), Phys. Rev. Lett. 72, 2332, (1994).

[13] S. Kuhlmann, private communication.

[14] A. Donnachie and P.V. Landshoff, Nucl. Phys. B244, 322 (1984).

[15] A. Donnachie and P.V. Landshoff, Nucl. Phys. B303, 634 (1988).

[16] P.V. Landshoff, private communication.

[17] E.L. Berger, J.C. Collins, D.E. Soper and G. Sterman, Nucl. Phys. B286, 704 (1987).

[18] R.D. Field and G. Fox, Nucl. Phys. B80, 367 (1974).

[19] A.B. Kaidalov, Phys. Rep. 50, 157 (1979).

[20] S.J. Brodsky, L. Frankfurt, J.F. Gunion, A.H. Mueller and M. Strikman, Phys. Rev. D50, 3134 (1994).

[21] H1 collaboration: A. De Roeck, invited talk at the Europhysics Conference on HEP, Marseille 1993 and DESY 94-005; and J.B. Dainton, invited talked at the XVI International Symposium on Lepton-Photon Interactions, Cornell 1993 and RAL-94-012.

[22] CDF Collaboration (F. Abe et al.), "Measurement of $\bar{p} p$ Single Diffraction Dissociation at $\sqrt{s}=546$ and $1800 \mathrm{GeV}$ ", FNAL preprint 93-233E.

[23] J. Orear, in Proceedings of Workshop on Small- $x$ and Diffractive Physics at the Tevatron, Fermilab, 16-18 Sep. 1992. 
[24] G.A. Schuler and T. Sjöstrand, Phys. Rev. D49, 2257 (1994).

[25] UA4 collaboration (M. Bozzo et al.), Phys. Lett. 155B, 197 (1985); Phys. Lett. 171B, 142 (1986).

[26] E.A. Kuraev, L.N. Lipatov and V.S. Fadin, Sov. Phys. JETP 44, 443 (1976), Sov. Phys. JETP 45, 199 (1977); Ya. Ya. Balitsky and L.N. Lipatov, Sov. J. Nucl. Phys. 28, 822 (1978).

[27] M.G. Sotiropoulos and G. Sterman, Nucl. Phys. B425, 489 (1994).

[28] C. DeTar, S.D. Ellis and P.V. Landshoff, Nucl. Phys. B87, 176 (1975).

[29] J.L. Cardy and G. Winbow, Phys. Lett. 52B, 95 (1974).

[30] J.C. Collins, D.E. Soper and G. Sterman, Nucl. Phys. B261, 104 (1985) and B308, 833 (1988); G. Bodwin, Phys. Rev. D31, 2616 (1985) and D34, 3932 (1986). For a review see J.C. Collins, D.E. Soper and G. Sterman, in "Perturbative QCD" (A.H. Mueller, ed.) (World Scientific, Singapore, 1989).

[31] A. Donnachie and P.V. Landshoff, Phys. Lett. 285B, 172 (1992).

[32] N.N. Nikolaev and B.G. Zakharov, in Ref. [10.

[33] F.V. Tkachov, Int. J. Mod. Phys. A8, 2047 (1993).

[34] UA4 Collaboration, M. Bozzo et al., Phys. Lett. 136B, 217 (1984); UA4 Collaboration, D. Bernard et al., Phys. Lett. 186B, 227 (1987).

[35] J. Pumplin, Phys. Rev. D47, 4820 (1993).

[36] F. Jacquet and A. Blondel, in Proc. of the study for an ep facility in Europe, ed. U. Amaldi, DESY 79/48 (1979) pp. 391-394.

[37] F. Ravndal, Phys. Lett. 43B, 301 (1973).

[38] R.L. Kingsley, Phys. Rev. D10, 1580 (1974).

[39] R. Meng, F.I. Olness and D.E. Soper, Nucl. Phys. B371, 79 (1992). 


\section{FIGURES}

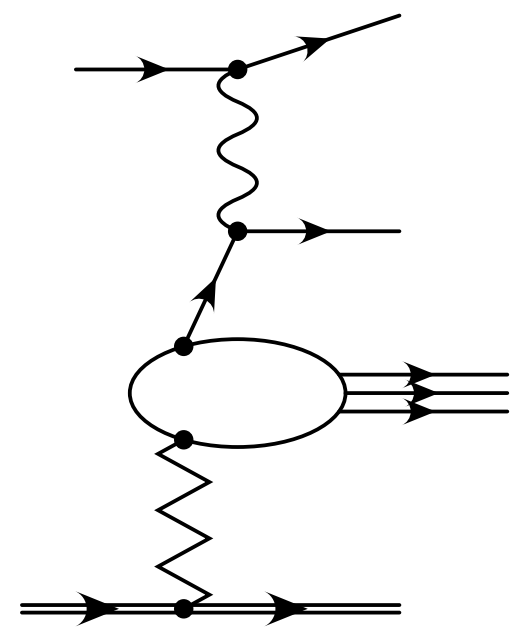

(a)

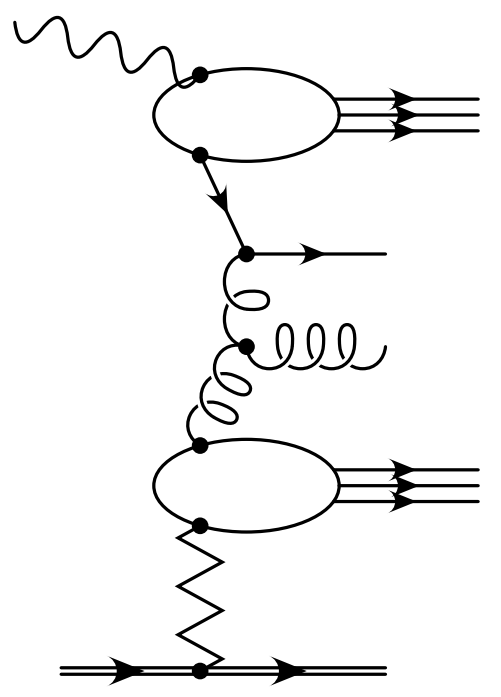

(c)

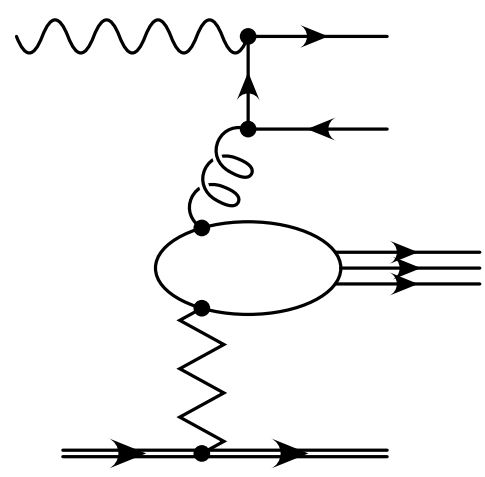

(b)

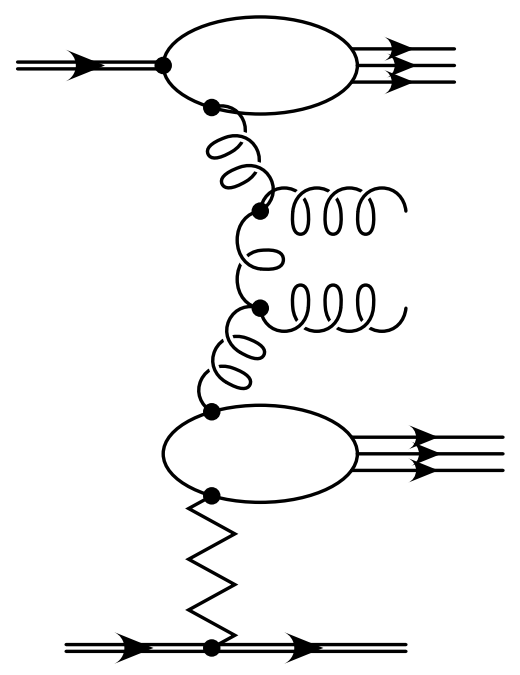

(d)

FIG. 1. The Ingelman-Schlein model for hard diffractive scattering, for the following processes: (a) electro-production, (b) direct and (c) resolved photo-production of jets, and (d) hadro-production of jets. 


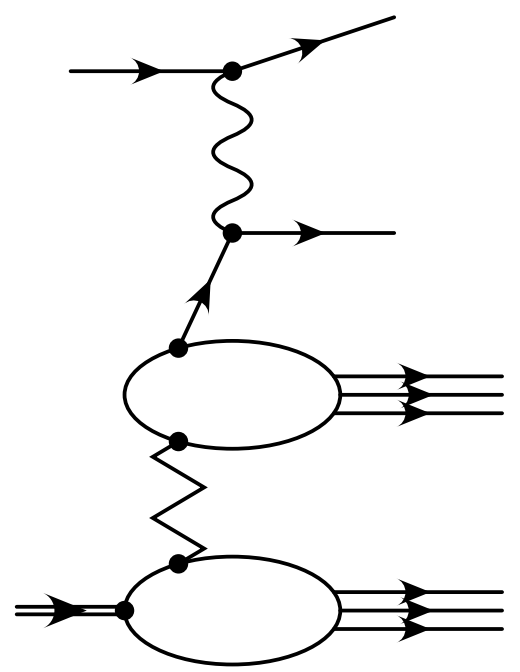

FIG. 2. Regge diagram for hard diffractive DIS with low mass excitation of proton.

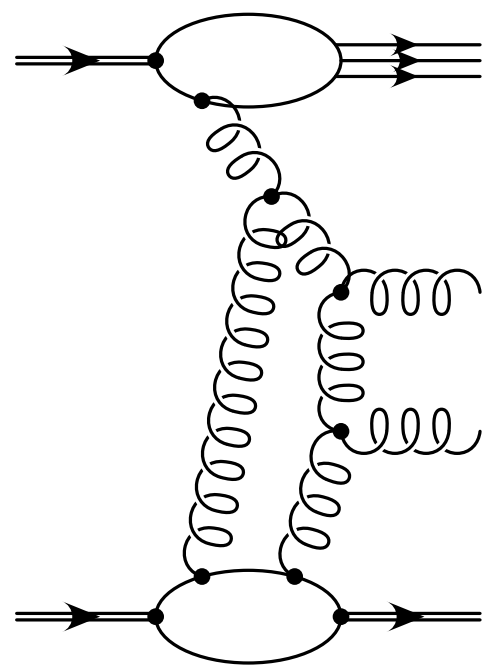

FIG. 3. Example of low-order graph for model of Collins, Frankfurt and Strikman [3].

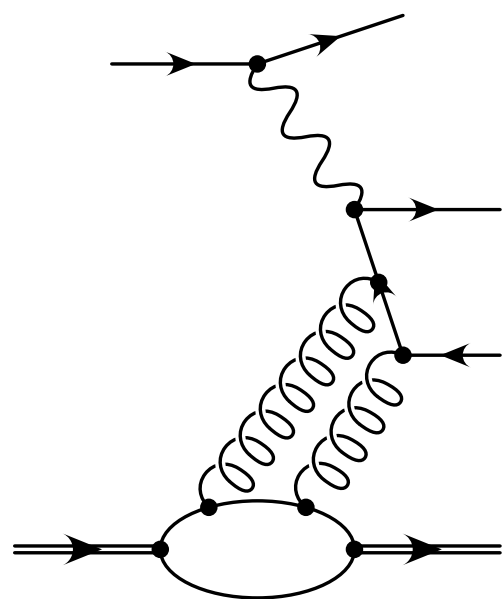

FIG. 4. Graph for coherent pomeron in deep inelastic scattering. 


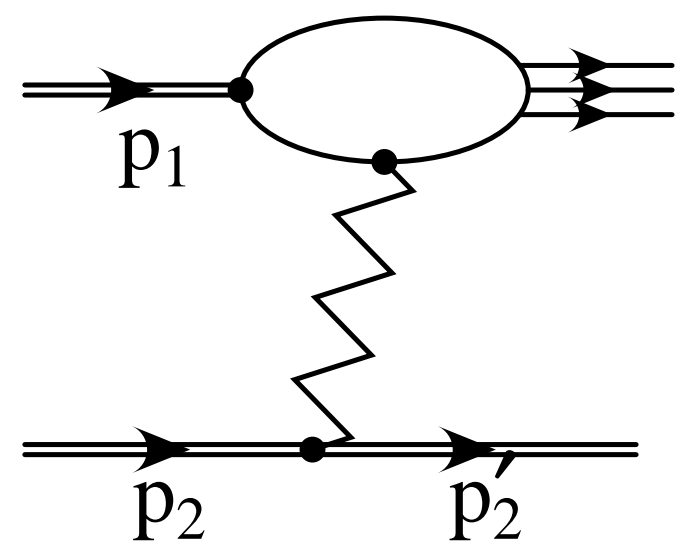

FIG. 5. Reaction used in definition of light-cone coordinates.

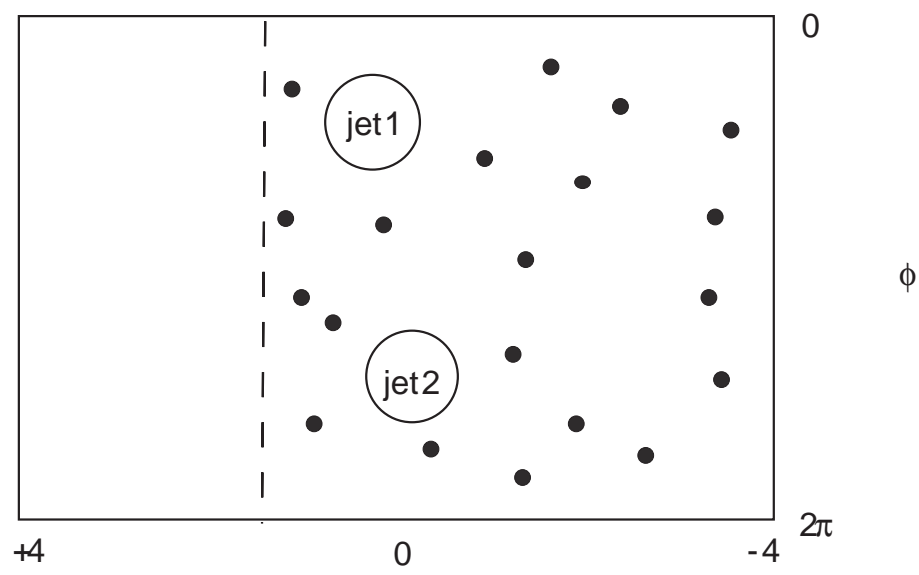

$\eta$

FIG. 6. $\eta$ - $\phi$ plot for the hard diffractive production of 2 jets. 


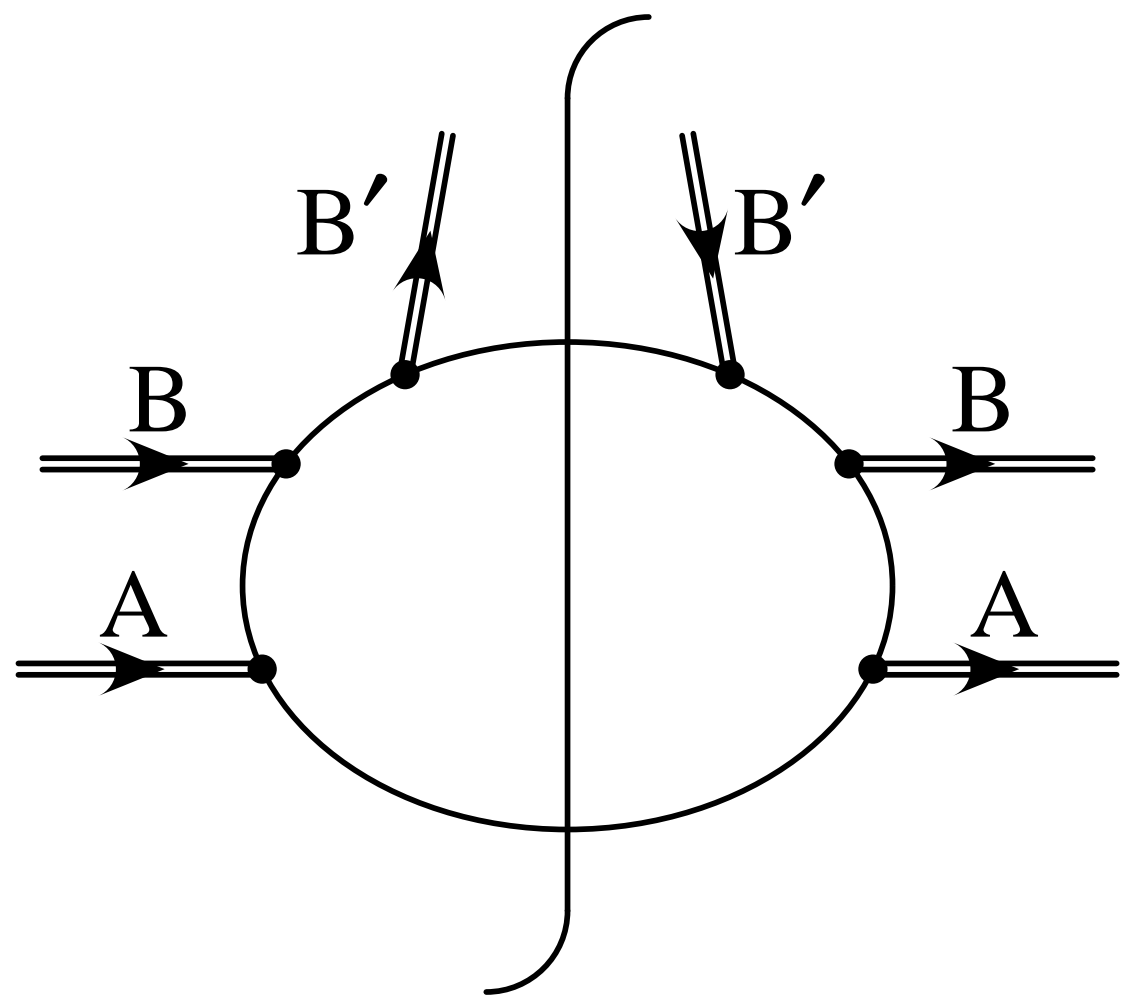

FIG. 7. Diffractive scattering is given by this discontinuity of a six point function. 
This figure "fig1-1.png" is available in "png" format from: http://arxiv.org/ps/hep-ph/9406255v3 
This figure "fig2-1.png" is available in "png" format from: http://arxiv.org/ps/hep-ph/9406255v3 
This figure "fig1-2.png" is available in "png" format from: http://arxiv.org/ps/hep-ph/9406255v3 
This figure "fig2-2.png" is available in "png" format from: http://arxiv.org/ps/hep-ph/9406255v3 
This figure "fig1-3.png" is available in "png" format from: http://arxiv.org/ps/hep-ph/9406255v3 
This figure "fig2-3.png" is available in "png" format from: http://arxiv.org/ps/hep-ph/9406255v3 
This figure "fig1-4.png" is available in "png" format from: http://arxiv.org/ps/hep-ph/9406255v3 
This figure "fig2-4.png" is available in "png" format from: http://arxiv.org/ps/hep-ph/9406255v3 
This figure "fig1-5.png" is available in "png" format from: http://arxiv.org/ps/hep-ph/9406255v3 